\title{
Observations on the Effects of a Chromosome Duplication in Aspergillus nidulans
}

\author{
By B. W. BAINBRIDGE* AND J. A. ROPER \\ Department of Genetics, University of Sheffield
}

(Received 4 October 1965)

\begin{abstract}
SUMMARY
During a study of genetic factors affecting the morphology of Aspergillus nidulans a novel and characteristic variant was observed among the progeny of crosses involving certain morphologically normal parents. This type, which had a diminished linear growth rate, segregated in constant proportion; it was designated 'crinkled'. It carried a duplication for a chromosome segment as a result of an unequal translocation in one parent. Crinkled colonies showed vegetative instability and sectored types which, in varying degree, approached normal morphology and growth rate. These revertants probably arose by loss of a variable part of the chromosome segment carried in duplicate. The loss occurred from either the translocated or untranslocated segment.
\end{abstract}

\section{INTRODUCTION}

During a study of genetic factors affecting morphology in Aspergillus nidulans a novel and characteristic variant, designated 'crinkled' ( $c r)$; was observed; $c r$ types posed two main problems. They arose regularly and in constant proportion from crosses of particular parents which had normal morphology. Furthermore, or colonies showed vegetative instability in giving sectors which, in varying degree, approached normal morphology and growth rate. The present paper describes an investigation into the genetic determination of $c r$ and the nature of its instability.

\section{METHODS}

The general techniques used in this work were those of Pontecorvo et al. (1953). Incubation was at $37^{\circ}$.

Media. Minimal medium (MM), Czapek-Dox with $1 \%$ glucose. Complete medium (CM), a complex medium containing yeast extract, hydrolysed casein, hydrolysed nucleic acid, vitamins, etc. Solid media contained $2 \%$ agar.

Organisms. Aspergillus nidulans strains were maintained on CM. Details of mutant alleles, and their locations, are given by Pontecorvo et al. (1953), Roper \& Käfer (1957), Käfer (1958) and Warr \& Roper (1965). Käfer (1961, 1962a, $b$, 1965) described the chromosome translocations in many of the currently used strains.

The mutants of main importance in this work were: $y$, yellow conidia; cha, chartreuse conidia; $w 2$ and $w 3$, white conidia; $a d 3$ and $a d 20$, arg1, bi1, cys2, lys5,

* Present address: Department of Microbiology, Queen Elizabeth College, Campden Hill Road, London, W. 8. 
nic2 and nic8, paba6, phen2, pro1, pyro4 and pyro12, ribol and ribo2, s1, s3 and s12, growth requirement, respectively, for adenine, arginine, biotin, cystine, lysine, nicotinic acid, $p$-aminobenzoic acid, phenylalanine, proline, pyridoxin, riboflavine and sulphite; su1-ad20, suppressor of $a d 20 ; A c r 1$, resistance to acriflavine. Translocations are designated by the symbol (T) after the genotype.

Genetic analysis. The general techniques of analysis used were those of Pontecorvo et al. (1953) with modified techniques for ascus dissection (Bainbridge, 1964) and for certain crosses (Bainbridge, 1965).

\section{RESULTS}

\section{The segregation of crinkled}

Table 1 shows the segregations from a number of crosses between particular parents with normal morphology. The $c r$ segregants had a characteristic phenotype (Pl.1, fig. 1) and a diminished linear growth rate; $c r$ segregants from the five crosses were phenotypically indistinguishable, suggesting a common mode of origin. However, in case they differed in origin, $c r$ segregants were numbered consecutively at isolation.

Table 1. Aspergillus nidulans: segregation of crinkled from crosses between morphologically normal parents

\begin{tabular}{|c|c|c|}
\hline \multirow[b]{2}{*}{ Parents } & \multicolumn{2}{|c|}{ Segregants } \\
\hline & Normal & Crinkled \\
\hline $1 ;$ ro3; cys2 $2 y ; a d 8 \mathrm{~s} 1$ & 898 & 193 \\
\hline $1 ; w 3 ; c y s 2 \times$ ribol $y ;$ nic8 & 192 & 98 \\
\hline $\begin{array}{l}\text { bi1; w3 } \text { su1-ad20 y ad20; Acr1; phen2; pyro4; lys5; } \\
\text { s3; nic8; ribo2 }\end{array}$ & 250 & 108 \\
\hline $1 ;$ w3; pyro12 ×s12; pyro4; nic2 & 294 & 148 \\
\hline i1; w3; pyro4 $\times$ ribol $y ;$ nic8 & 884 & 424 \\
\hline
\end{tabular}

* $\chi^{2}$ test for fit to 2:1 ratio of normal: crinkled. Results from the last two crosses were kindly supplied by Dr J. R. Warr.

The segregation data were statistically inconsistent with $1: 1$ or $3: 1$ ratios but showed good agreement with a ratio of 2 normal: $1 \mathrm{cr}$. This suggested the possibility of two freely-recombining interacting genes in which one recombinant class was nonviable and the other $\mathrm{cr}$. Ascus analysis was undertaken on only a small scale. However, the results indicated inviability of a type which could not be ascribed readily either to technique or to chance inviability of a proportion of ascospores. From the cross, bi1 $\times y$; ad3; s1 cys2, four classes of asci were found in the 10 asci analysed. Five asci were of type $I$ with 4 spore pairs giving morphologically normal colonies; 1 type II ascus with $2 c r, 2$ nonviable spore pairs; 2 type III asci with 2 normal, $1 \mathrm{cr}, 1$ nonviable spore pairs and 2 type IV asci with 4 nonviable spore pairs. These segregation patterns corresponded to asci showing the following spore sizes: I, 8 normal; II, 4 normal, 4 small; III, 6 normal, 2 small, and IV, 8 small. Overall ascospore viability, both from ascus analysis and random spore platings, was about $60 \%$.

Two freely-recombining genes with the interactions outlined above would yield asci of types, I, II and III but type IV would not be expected and it was necessary 
to seek an explanation of $c r$ in other terms. The data of Käfer showed that in all the crosses of Table 1 one or other parent carried a chromosome translocation involving linkage groups III and VIII and it seemed reasonable to explore the possible association of this translocation and $c r$. Pritchard (1960; and personal communication) had already reported a strain of Aspergillus nidulans carrying a chromosome duplication and with a morphology somewhat similar to $\mathrm{cr}$. Furthermore, from a cross in Neurospora crassa involving an unequal translocation in one parent, McClintock (1945) reported ascus analysis data similar in patterns of defective spores to those given above.

Many crosses were examined for the segregation of $\mathrm{cr}$ but consideration will be given here only to those crosses involving parents of known status in respect of the III-VIII translocation. There were 28 crosses involving 27 different, morphologically normal parent strains. Those crosses in which both parents or neither parent carried the translocation did not yield $c r$ segregants; all crosses in which only one parent carried the translocation did yield $c r$ among the progeny.

The segregation data could be accommodated if the translocation were unequal. A cross heterozygous for this translocation would yield $25 \%$ of the progeny with a deficiency and $25 \%$ with a duplication. The former would certainly be nonviable and the latter, presumably, cr. It remained to test this idea and to determine the direction of the III-VIII translocation. Mitotic haploidization is used to detect heterozygous translocations (Käfer, 1962b). During haploidization of diploid nuclei of Aspergillus nidulans there is no crossing-over and markers on the two linkage groups involved in a translocation show complete linkage, in contrast to the free recombination expected in translocation-free diploids. However, this first analysis did not reveal either the direction or extent of the translocation.

Table 2. Aspergillus nidulans: segregations from crosses involving a crinkled parent

\begin{tabular}{|c|c|c|c|}
\hline \multirow[b]{2}{*}{ Cross } & \multicolumn{2}{|c|}{ Segregants } & \multirow[b]{2}{*}{$\mathbf{P} *$} \\
\hline & Normal & Crinkled & \\
\hline $\operatorname{cr14} y ; a d 3 \times b i 1 ; w 3 ; c y s 2(\mathrm{~T})$ & 73 & 74 & $>0.9$ \\
\hline cr15 prol $y \times b i 1 ;$ w3; pyro12 $(\mathrm{T})$ & 48 & 54 & 0.5 \\
\hline$c r 16 y \times b i 1 ; w 3 ; c y s 2(\mathrm{~T})$ & 102 & 97 & 0.7 \\
\hline cr4 bi1 $\times y ;$ w2; arg1 & 134 & 143 & $\mathbf{0 . 5}$ \\
\hline
\end{tabular}

(T) indicates a strain carrying the III-VIII translocation.

* $\chi^{2}$ test for agreement with $1: 1$ ratio.

As a first step towards testing the idea of an association of $\mathrm{cr}$ and a chromosome duplication, or segregants from various crosses were backcrossed to strains of normal morphology with or without the III-VIII translocation. In both types of cross the only chromosomal difference between the parental strains of each cross was the suspected presence of a duplicated chromosome segment. A 1:1 ratio of $\mathrm{cr}$ type to normal was therefore expected in the progeny. Table 2 shows a selection of the results obtained and these are all consistent with the expected ratio. Certain anomalous backcross results, obtained in other crosses, are discussed later. 


\section{Anomalous segregations of nutritional markers among cr progeny}

Normal and $\mathrm{cr}$ progeny from various crosses of normal parents were classified for the segregation of a number of nutritional markers. Allele ratios for both morphological classes were 1:1 except in the case of cys2 which is located on the right arm of linkage group III. From a cross of an untranslocated strain to a strain carrying cys 2 and the III-VIII translocation, normal and $c r$ segregants were tested for cystine requirement. This marker gave a 1:1 segregation among morphologically normal progeny but of $70 \mathrm{cr}$ segregants only 6 required cystine. This suggested that

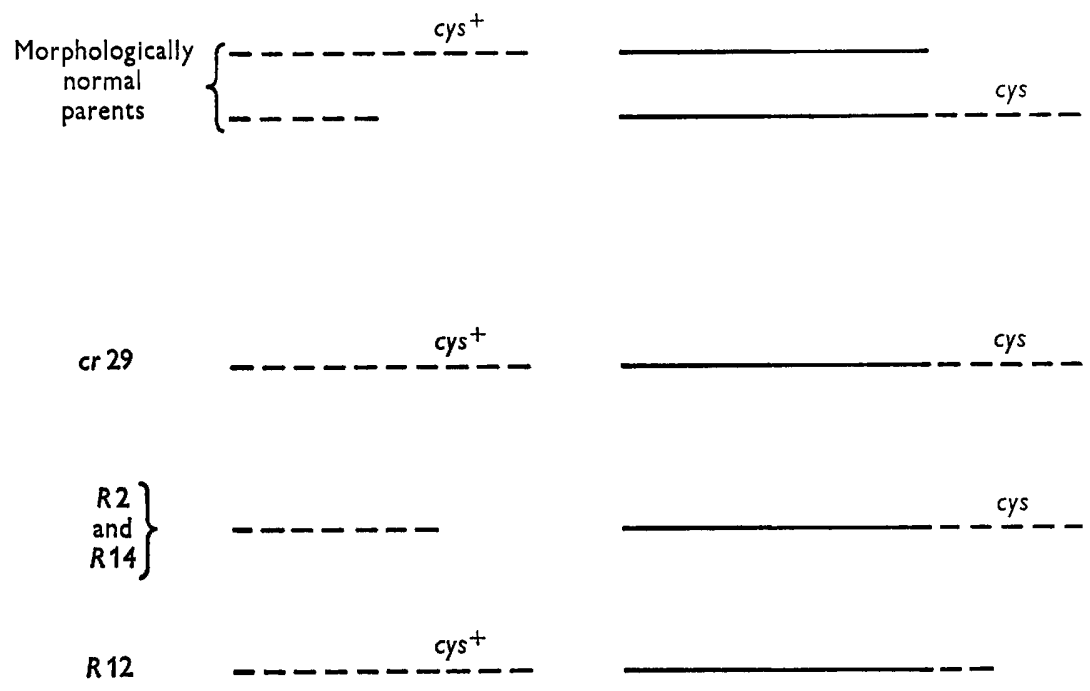

Fig. 1. Aspergillus nidulans. A diagrammatic representation of the origin of $\mathrm{cr}$ and revertants of $c r$. Broken and unbroken lines represent chromosomes III and VIII respectively.

the second parent in the above cross had a translocation to chromosome VIII of the segment of chromosome III which carries cys2. The $c r$ segregants would then carry this segment of chromosome III in duplicate (Fig. 1). Most cr segregants would be cys2/cys2 ${ }^{+}$and only those which resulted from crossing-over between cys 2 and the point of translocation, followed by appropriate segregation, would be cys2/cys2. The alternative, a translocation from chromosome VIII to chromosome III, would have given $c r$ types with duplication of a segment of chromosome VIII. In this case at least $50 \%$ of $c r$ segregants would have required cystine. The frequency of $c r$, cystine-requiring segregants gave a measure of the extent of the translocation and indicated that cys2 shows about $17 \%$ recombination with the point of break.

A number of cystine-independent $c r$ segregants were selected from crosses involving the marker cys2. Each was crossed to a morphologically normal, cystineindependent strain with the III-VIII translocation. Several crosses gave no segregants requiring cystine and the $c r$ parents in these were presumably $c y s 2^{+} / c y s 2^{+}$. Two crosses yielded cystine requirers. Of 25 normal segregants from the cross, cr15 pro1 $y \times b i 1 ; w 3 ;$ pyro12, 12 required and 13 were independent of cystine. From the cross, $c r 29$ bi1; w2; $\arg 1 \times a d 3$, both crinkled and normal segregants were 
classified. The normal segregants were 23 independent and 14 requirers while of 29 crinkled segregants tested only 1 required cystine. These crosses showed unequivocally that certain $c r$ strains were heterozygous $c y s 2 / c y s 2^{+}$and carried, therefore, a duplication for a segment of chromosome III.

\section{Instability of cr strains}

Table 3 lists further segregations from backcrosses of $c r$ strains and the results are in sharp contrast to those of Table 2 . These results indicated possible instability of $c r$ strains; they were later explained in terms of vegetative instability.

Table 3. Aspergillus nidulans: anomalous results from crosses involving a crinkled parent

\begin{tabular}{|c|c|c|c|}
\hline \multirow[b]{2}{*}{ Cross } & \multicolumn{2}{|c|}{ Segregants } & \multirow[b]{2}{*}{ P* } \\
\hline & Normal & Crinkled & \\
\hline$c r 6 b i 1 ; a d 3 \times b i 1 ; w 3(\mathrm{~T})$ & $\mathbf{2 8 4}$ & 137 & $\ll 0.001$ \\
\hline$c r 4 b i 1 \times b i 1 ; z 03 ; c y s 2(\mathrm{~T})$ & 515 & 236 & $\ll 0.001$ \\
\hline cr4 bi1 × pro1 paba6 y† & 118 & 10 & $\ll 0.001$ \\
\hline cr4 bi1 xprol paba6 $y \dagger$ & 308 & 12 & $\ll 0.001$ \\
\hline cr10 bi $1 ; w 3$ ad $3 \times$ prol paba $6 y$ & 149 & 71 & $\ll 0.001$ \\
\hline
\end{tabular}

(T) indicates a strain carrying the III-VIII translocation.

* $\chi^{2}$ test for agreement with $1: 1$ ratio.

$\uparrow$ Results from different individual perithecia.

Three $c r$ strains were selected from the cross $b i 1 ;$ r $w$; cys 2 (T) $\times y$; ad5; s1. Each was inoculated at a single point in the centre of several plates of CM. After 10-days incubation more than half of the colonies showed 'revertant' sectors (Pl. 1, fig. 2). The $c r$ parents had a linear growth rate about $53 \%$ that of wild type and the revertant sectors ranged between 58 and $97 \%$ of wild type. All the revertant sectors could still be distinguished from the morphologically normal parents in showing some degree of the general phenotypic features of $\mathrm{cr}$. It seemed likely that reversion could be explained in one of two ways. Revertants might arise from mutations which suppressed, in varying degree, the morphological effects of the duplication. Alternatively, there might have been loss at mitosis of a part, presumably a variable part, of one of the chromosome segments carried in duplicate.

A number of $c r$ segregants were selected from the cross bil; w3; cys $2(\mathrm{~T}) \times y$; w2; arg1. These segregants, and revertants derived from them, were classified for cystine requirement with the results shown in Table 4. cr strains 34, 35 and 36 are presumably cys2/cys2 in genotype; the genotypes of strains 25, 26, 28, 30 and 33 cannot be determined from the data available; 23,29 and 31 showed vegetative segregation indicating a genotype cys2/cys2 ${ }^{+}$. These results confirmed the duplication, in $c r$ strains, of a segment of chromosome III and they also strongly indicated the process of reversion. It seemed highly probable that the revertants arose by loss, at mitosis, of part or all of the duplicated chromosome segment. Furthermore, cr31 and cr29 segregated both cystine-requiring and cystine-independent revertants indicating that the proposed chromosome loss could take place from either the translocated or untranslocated segment.

In an attempt to confirm this last point, three of the revertants from cr29 were 
crossed to morphologically normal strains with or without the III-VIII translocation. The results are shown in Table 5; no distinction was made in classification between segregants with revertant and those with wild type morphology. Revertants 2 and 14 gave segregations consistent with a 2:1 ratio when crossed with an untranslocated strain but not when crossed with a strain carrying the translocation. The reverse situation obtained for revertant 12. These results could be explained in the terms shown diagrammatically in Fig. 1. cr29 is shown with a duplication

Table 4. Aspergillus nidulans: characterization of cr segregants and their revertants for cystine requirement

$\begin{array}{cccc}\begin{array}{c}\text { Parent } \\ \text { cr } \text { strain }\end{array} & \overbrace{\text { Parent }}^{\begin{array}{c}\text { Requirement for }(-) \text { /independence } \\ \text { of }(+) \text { cystine }\end{array}} & \overbrace{+}^{\text {Revertants }} \\ \mathbf{3 4} & - & 0 & - \\ \mathbf{3 5} & - & 0 & \mathbf{1} \\ \mathbf{3 6} & - & \mathbf{0} & \mathbf{2} \\ \mathbf{2 3} & + & \mathbf{0} & \mathbf{1} \\ \mathbf{2 5} & + & \mathbf{1} & \mathbf{0} \\ \mathbf{2 6} & + & \mathbf{3} & \mathbf{0} \\ \mathbf{2 8} & + & \mathbf{1} & \mathbf{0} \\ \mathbf{2 9} & + & \mathbf{1 1} & \mathbf{5} \\ \mathbf{3 0} & + & \mathbf{2} & \mathbf{0} \\ \mathbf{3 1} & + & \mathbf{1} & \mathbf{1} \\ \mathbf{3 3} & + & \mathbf{2} & \mathbf{0}\end{array}$

The cr parents were segregants from the cross bi1; w3; cys2 (T) $\times y$; wo2; $\operatorname{arg1}$.

Table 5. Aspergillus nidulans: segregations from crosses of $\mathrm{cr}$ revertants

\begin{tabular}{|c|c|c|c|c|c|c|}
\hline \multirow[b]{2}{*}{ Revertant* } & \multicolumn{2}{|c|}{ Cross to $y ; a d 3(\mathrm{~T})$} & \multirow[b]{2}{*}{$P \ddagger$} & \multicolumn{2}{|c|}{ Cross to ribol $y ;$ nic8 } & \multirow[b]{2}{*}{$\mathbf{P} \ddagger$} \\
\hline & Normal $\dagger$ & Crinkled & & Normal $\dagger$ & Crinkled & \\
\hline 2 & 165 & 10 & $\ll 0.001$ & 226 & 114 & $>0.9$ \\
\hline 12 & 104 & 51 & $0 \cdot 9$ & 199 & $\mathbf{3}$ & $\ll 0.001$ \\
\hline 14 & 221 & 7 & $\ll 0.001$ & 202 & 125 & 0.05 \\
\hline
\end{tabular}

* Spontaneous revertants from cr29 bi1; ro2; cys2/cys2+; arg1.

$\dagger$ Revertants and normal were similar in morphology and were not distinguished in classification. $\ddagger \chi^{2}$ test for agreement with 2:1 ratio of normal: crinkled.

of a segment of chromosome III. It was certainly heterozygous cys2/cys2+ and it was highly likely, from consideration of its pedigree, that $c y s^{+}$was carried on the untranslocated segment. In crosses with translocation-free strains, $R 2$ and $R 14$ behaved like morphologically normal strains carrying the III-VIII translocation; they yielded a 2:1 ratio of normal:cr. They had presumably lost, from the previously intact chromosome III, most of that segment of III which was also represented in the translocation to VIII. However, they could not have lost the whole of the segment. From crosses with normal strains carrying the translocation they yielded a low frequency of $\mathrm{cr}$ segregants and this would not have been the case for two morphologically normal translocated parents. The low proportion of $\mathrm{cr}$ could 
arise by crossing-over in, and appropriate segregation from, the quadrivalent formed at meiosis. A similar argument could be applied to $R 12$ except that in this case the behaviour of the revertant approached that of an untranslocated normal strain; it yielded very low frequencies of $c r$ segregants in a cross with a translocationfree strain and gave a 2:1 ratio normal:cr in a cross with a normal strain carrying the translocation.

It was suggested earlier that mutations at suppressor loci might be invoked to explain reversion. It could be supposed that such mutations would completely or partially suppress the phenotypic effects of the duplications. The results of Tables 4 and 5 excluded the possibility of suppressors which recombined freely with the point of translocation. To maintain this possibility, in view of the vegetative segregation shown in Table 4, it would have been necessary to suggest that each suppressor was specific for one or other of the duplicated segments; this seemed very unlikely. Moreover, certain crosses of revertants (Table 5) yielded only a low frequency of $\mathrm{cr}$ segregants; a freely-recombining suppressor would have yielded a far higher frequency. However, there remained the possibility, admittedly remote, that reversion might be explained not by chromosome loss but by mutations at suppressor loci linked to one or other duplicated chromosome III segment. Such mutations would have to suppress both the linked cys allele and the phenotypic effects of the segment of chromosome distal to the point of mutation. R14 required cystine; on the basis of this argument it would have carried a suppressed cys $2^{+}$ allele which it should have been possible to reveal in appropriate crosses. R14 was crossed with bi1; cys2; lys5; cha and a total of 20,000 ascospores, of hybrid origin, plated on MM supplemented with all requirements except cystine. From platings at various concentrations no cystine-independent segregants were recovered.

\section{DISCUSSION}

The observed morphological effects of a chromosome duplication were not surprising since such effects are well known in a wide variety of organisms. However, the vegetative instability was unexpected. So far we have observed this instability through the sectors formed from $\mathrm{cr}$ colonies. Our data do not permit a calculation of the reversion rate. Such a calculation could be based on the frequency of revertants obtained by plating conidia from $\mathrm{cr}$ strains. Both from such platings, and from the frequency of sectors yielded by $\mathrm{cr}$ colonies, it may also be possible to investigate genetic and environmental factors which influence the reversion rate. For the present we can only say that the frequency of spontaneous reversion of $c r$ was far greater than that of gene-determined slow-growing variants.

Reversion could best be explained by spontaneous chromosome or chromatid breakage, followed by loss of the fragment at mitosis. It could also be explained by an error in replication which resulted in a break in the continuity of the chromosomal material. We were fortunate in having a system which selects for revertant events but our results do not yet permit distinction between these two possible modes of origin.

When this work was done there were few known markers on the relevant segment of chromosome III. A search is now being made for further markers in this segment and for a similar situation involving other well-marked chromosome segments. It 
should then be possible to define the positions of chromosome loss more precisely. Such a system might lend itself to a study of chromosome structure, especially if it were possible to find agents or conditions which selectively produced losses in particular regions of the duplicated segment. The positions of loss would correspond to points sensitive either to breakage or to the interruption of replication.

The authors are grateful to Dr A. J. Clutterbuck and Dr J. R. Warr for valuable discussions. They are particularly indebted to Dr E. Käfer-Boothroyd who made strains and unpublished data freely available; without this help the work could not have been completed. An A.R.C. Postgraduate Studentship to one of us (B. W. B) is gratefully acknowledged.

\section{REFERENCES}

BAINBRIDGE, B. W. (1964). Genetic factors affecting the morphology of Aspergillus nidulans. Ph.D. thesis, University of Sheffield.

Bainbridge, B. W. (1965). The arginine crossing technique. Aspergillus Neres Letter, 6, 8. KäFER, E. (1958). An 8-chromosome map of Aspergillus nidulans. Advanc. Genet. 9, 105. KäFER, E. (1961). Translocations in Aspergillus nidulans. Aspergillus Newes Letter, $2,10$. KÄFER, E. (1962a). Translocations in Aspergillus nidulans. Aspergillus News Letter, 3, 3. KÄFER, E. (1962b). Translocations in stock strains of Aspergillus nidulans. Genetica, 33, 59. KäFER, E. (1965). Origins of translocations in Aspergillus nidulans. Genetics, 52, 217.

McClintock, B. (1945). Neurospora. I. Preliminary observations of the chromosomes of Neurospora crassa. Am. J. Bot. 32, 671.

Pontecorvo, G., Roper, J. A., Hemmons, L. M., MacDonald, K. D. \& Bufton, A. W. J. (1953). The genetics of Aspergillus nidulans. Advanc. Genet. 5, 141.

Pritchard, R. H. (1960). The bearing of recombination analysis at high resolution on genetic fine structure in Aspergillus nidulans and the mechanism of recombination in higher organisms. In Microbial Genetics. Symp. Soc. gen. Microbiol. 10, 155.

ROPER, J. A. \& KäFER, E. (1957). Acriflavine-resistant mutants of Aspergillus nidulans. J. gen. Microbiol. 16, 660.

WARR, J. R. \& Roper, J. A. (1965). Resistance to various inhibitors in Aspergillus nidulans. J. gen. Microbiol. 40, 273.

\section{EXPLANATION OF PLATE}

Fig. 1. A crinkled colony and, for comparison, the edge of a normal colony. $\times 3$.

Fig. 2. A crinkled colony with revertant sectors. $\times 1$. 
Journal of General Microbiology, Vol. 42, No. 3

Plate 1

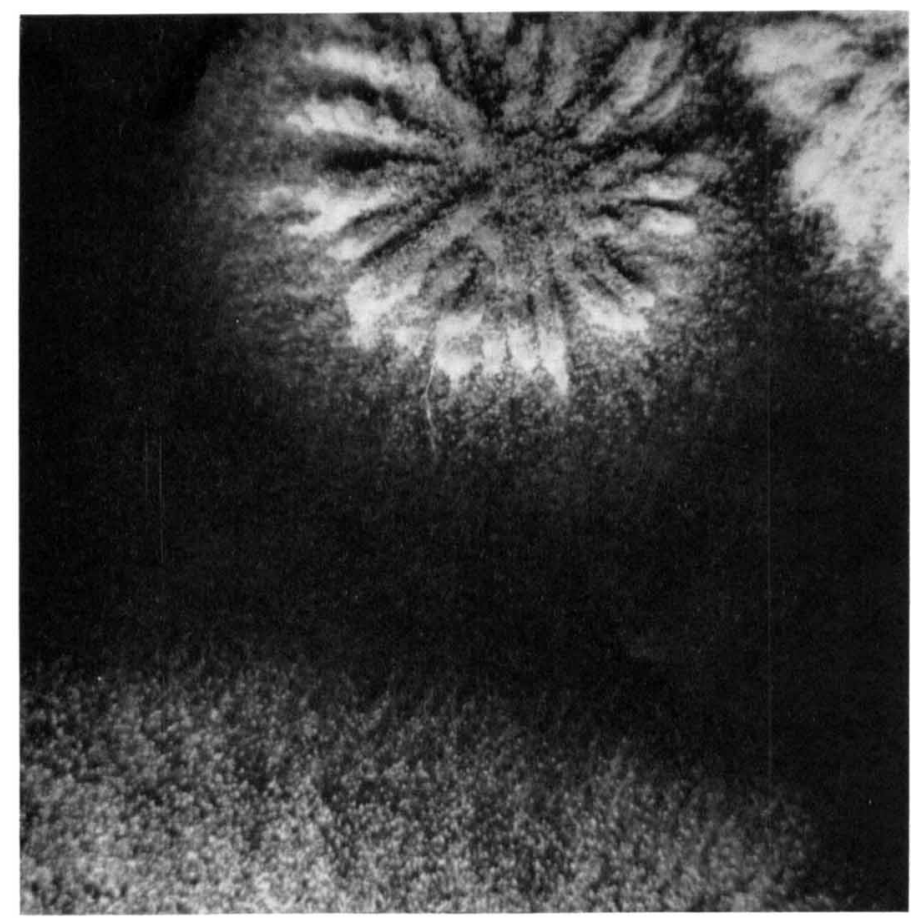

Fig. 1

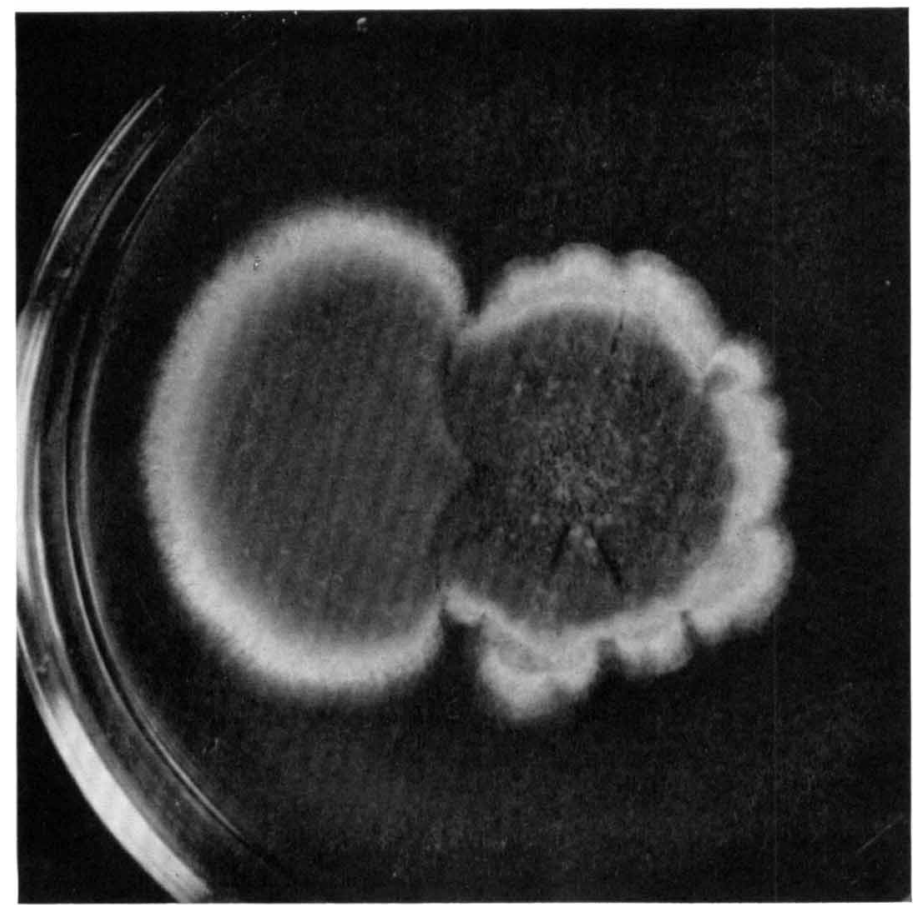

Fig. 2

B. W. BAINBRIDGE AND J. A. ROPER

(Facing $p .424$ ) 